International Mathematical Forum, Vol. 9, 2014, no. 17, 797 - 801

HIKARI Ltd, www.m-hikari.com

http://dx.doi.org/10.12988/imf.2014.4478

\title{
On Certain Sums Extended over Prime Factors
}

\author{
Rafael Jakimczuk \\ División Matemática, Universidad Nacional de Luján \\ Buenos Aires, Argentina
}

Copyright (c) 2014 Rafael Jakimczuk. This is an open access article distributed under the Creative Commons Attribution License, which permits unrestricted use, distribution, and reproduction in any medium, provided the original work is properly cited.

Abstract
Let $P(n)$ be the greatest prime factor of a positive integer $n$. In this
note we obtain asymptotic formulae for the sums
$\sum_{k=1}^{n} \frac{P(k)^{m}}{k^{s}} \quad(s=0,1, \ldots, m)$,

where $m$ is an arbitrary but fixed positive integer.

We also obtain asymptotic formulae for the sum

$$
\sum_{k=1}^{n} \frac{p(k)^{m}}{k^{s}} \quad(s=0,1, \ldots, m)
$$

where $p(n)$ denotes the least prime factor of $n$.

Mathematics Subject Classification: 11A99, 11B99

Keywords: Sums over prime factors

\section{Introduction}

Let $P(n)$ be the greatest prime factor of a positive integer $n$ and let $m$ be an arbitrary but fixed positive integer. For completeness, let $P(1)=1$. Let us consider the $m+1$ sums

$$
F_{m}^{s}(n)=\sum_{k=1}^{n} \frac{P(k)^{m}}{k^{s}} \quad(s=0,1, \ldots, m) .
$$


The case $m=1$ and $s=0$ was studied by K. Alladi and P. Erdős [1]. These authors obtained the following asymptotic formula

$$
F_{1}^{0}(n)=\sum_{k=1}^{n} P(k) \sim \frac{\pi^{2}}{12} \frac{n^{2}}{\log n}=\frac{\zeta(2)}{2} \frac{n^{2}}{\log n},
$$

where $\zeta(s)$ denotes the Riemann's zeta function.

The case $m=1$ and $s=1$ was studied by J. Kemeny [3]. This author obtained the following asymptotic formula

$$
F_{1}^{1}(n)=\sum_{k=1}^{n} \frac{P(k)}{k} \sim \frac{\pi^{2}}{6} \frac{n}{\log n}=\zeta(2) \frac{n}{\log n} .
$$

J. Kemeny claims that formula (2) can be obtained from his formula (3). However, we shall see that formula (3) can be obtained from formula (2).

The case $s=0$ was studied by R. Jakimczuk [2]. This author obtained the following asymptotic formula

$$
F_{m}^{0}(n)=\sum_{k=1}^{n} P(k)^{m} \sim \frac{\zeta(m+1)}{m+1} \frac{n^{m+1}}{\log n} \quad(m \geq 1) .
$$

If $m=1$ then equation (4) becomes equation (2). In this note we study the more general sums (1).

Let $p(n)$ be the least prime factor of a positive integer $n$. In this note we also obtain asymptotic formulae for the sums

$$
f_{m}^{s}(n)=\sum_{k=1}^{n} \frac{p(k)^{m}}{k^{s}} \quad(s=0,1, \ldots, m),
$$

where $m$ is an arbitrary but fixed positive integer.

\section{Main Results}

Lemma 2.1 Let $\sum_{i=1}^{\infty} a_{i}$ and $\sum_{i=1}^{\infty} b_{i}$ be two series of positive terms such that $a_{i} \sim b_{i}$. If $\sum_{i=1}^{\infty} b_{i}$ is divergent then we have the following asymptotic formula

$$
\sum_{i=1}^{n} a_{i} \sim \sum_{i=1}^{n} b_{i}
$$

Proof. See for example ([4], page 332).

Theorem 2.2 Let $m$ be an arbitrary but fixed positive integer. We have the following asymptotic formulae

$$
F_{m}^{s}(n)=\sum_{k=1}^{n} \frac{P(k)^{m}}{k^{s}} \sim \frac{\zeta(m+1)}{m-s+1} \frac{n^{m-s+1}}{\log n} \quad(s=0,1, \ldots, m) .
$$


Proof. Let $m$ be a fixed positive integer. If $s=0$ then formula (5) is true (see formula (4)). Suppose that formula (5) is true for $s \in\{0, \ldots, m-1\}$. We shall prove that formula (5) is also true for $s+1$.

We have

$$
\begin{aligned}
F_{m}^{s+1}(n) & =F_{m}^{s}(1)+\frac{1}{2}\left(F_{m}^{s}(2)-F_{m}^{s}(1)\right)+\frac{1}{3}\left(F_{m}^{s}(3)-F_{m}^{s}(2)\right)+\cdots \\
& +\frac{1}{n}\left(F_{m}^{s}(n)-F_{m}^{s}(n-1)\right)=\left(1-\frac{1}{2}\right) F_{m}^{s}(1)+\left(\frac{1}{2}-\frac{1}{3}\right) F_{m}^{s}(2) \\
& +\left(\frac{1}{3}-\frac{1}{4}\right) F_{m}^{s}(3)+\cdots+\left(\frac{1}{n-1}-\frac{1}{n}\right) F_{m}^{s}(n-1)+\frac{1}{n} F_{m}^{s}(n) \\
& =\frac{1}{1.2} F_{m}^{s}(1)+\frac{1}{2.3} F_{m}^{s}(2)+\frac{1}{3.4} F_{m}^{s}(3)+\cdots+\frac{1}{(n-1) n} F_{m}^{s}(n-1) \\
& +\frac{1}{n} F_{m}^{s}(n)=\frac{1}{2} F_{m}^{s}(1)+\frac{1}{3} \frac{F_{m}^{s}(2)}{2}+\frac{1}{4} \frac{F_{m}^{s}(3)}{3}+\cdots+\frac{1}{n} \frac{F_{m}^{s}(n-1)}{n-1} \\
& +\frac{1}{n+1} \frac{F_{m}^{s}(n)}{n}+\frac{F_{m}^{s}(n)}{n+1} .
\end{aligned}
$$

That is,

$$
F_{m}^{s+1}(n)=\frac{1}{2} F_{m}^{s}(1)+\frac{1}{3} \frac{F_{m}^{s}(2)}{2}+\frac{1}{4} \frac{F_{m}^{s}(3)}{3}+\cdots+\frac{1}{n+1} \frac{F_{m}^{s}(n)}{n}+\frac{F_{m}^{s}(n)}{n+1}
$$

Now, we have (see (5))

$$
F_{m}^{s}(n)=f(n) \frac{\zeta(m+1)}{m-s+1} \frac{n^{m-s+1}}{\log n} \quad(n \geq 2),
$$

where $f(n) \rightarrow 1$.

Therefore

$$
\frac{F_{m}^{s}(n)}{n+1}=f(n) \frac{n}{n+1} \frac{\zeta(m+1)}{m-s+1} \frac{n^{m-s}}{\log n}=g(n) \frac{\zeta(m+1)}{m-s+1} \frac{n^{m-s}}{\log n} \quad(n \geq 2),
$$

where $g(n) \rightarrow 1$, and

$$
\begin{aligned}
& \frac{1}{i+1} \frac{F_{m}^{s}(i)}{i}=f(i) \frac{i}{i+1} \frac{\zeta(m+1)}{m-s+1} \frac{i^{m-s-1}}{\log i} \\
= & h(i) \frac{\zeta(m+1)}{m-s+1} \frac{i^{m-s-1}}{\log i} \quad(i \geq 2),
\end{aligned}
$$

where $h(i) \rightarrow 1$. That is,

$$
\frac{1}{i+1} \frac{F_{m}^{s}(i)}{i} \sim \frac{\zeta(m+1)}{m-s+1} \frac{i^{m-s-1}}{\log i} \quad(i \geq 2) .
$$


Consequently (9) and lemma 2.1 give

$$
\sum_{i=2}^{n} \frac{1}{i+1} \frac{F_{m}^{s}(i)}{i} \sim \sum_{i=2}^{n} \frac{\zeta(m+1)}{m-s+1} \frac{i^{m-s-1}}{\log i}
$$

We have (L'Hospital's rule)

$$
\lim _{x \rightarrow \infty} \frac{\int_{a}^{x} \frac{t^{m-s-1}}{\log t} d t}{\frac{x^{m-s}}{(m-s) \log x}}=\lim _{x \rightarrow \infty} \frac{\frac{x^{m-s-1}}{\log x}}{\frac{d}{d x}\left(\frac{x^{m-s}}{(m-s) \log x}\right)}=1 \quad(s=0, \ldots, m-1) .
$$

Note that if $s=0, \ldots, m-2$ then the function $\frac{x^{m-s-1}}{\log x}$ is strictly increasing from a certain $n_{0}$. Consequently (see (11))

$$
\begin{aligned}
\sum_{i=2}^{n} \frac{i^{m-s-1}}{\log i} & =\sum_{i=2}^{n_{0}-1} \frac{i^{m-s-1}}{\log i}+\int_{n_{0}}^{n} \frac{x^{m-s-1}}{\log x} d x \\
& +O\left(\frac{n^{m-s-1}}{\log n}\right) \sim \frac{n^{m-s}}{(m-s) \log n} .
\end{aligned}
$$

Since the left side of the equality is a sum of rectangles of basis 1 .

On the other hand if $s=m-1$ then the function $\frac{x^{m-s-1}}{\log x}$ is strictly decreasing. Consequently (see (11))

$$
\sum_{i=2}^{n} \frac{i^{m-s-1}}{\log i}=\int_{2}^{n} \frac{x^{m-s-1}}{\log x} d x+O(1) \sim \frac{n^{m-s}}{(m-s) \log n} .
$$

Since the left side of the equality is a sum of rectangles of basis 1 .

Equations (10), (12) and (13) give

$$
\sum_{i=2}^{n} \frac{1}{i+1} \frac{F_{m}^{s}(i)}{i}=k(n) \frac{\zeta(m+1)}{m-s+1} \frac{n^{m-s}}{(m-s) \log n}
$$

where $k(n) \rightarrow 1$.

Equations (6), (8) and (14) give

$$
\begin{aligned}
F_{m}^{s+1}(n) & =\frac{1}{2} F_{m}^{s}(1)+k(n) \frac{\zeta(m+1)}{m-s+1} \frac{n^{m-s}}{(m-s) \log n}+g(n) \frac{\zeta(m+1)}{m-s+1} \frac{n^{m-s}}{\log n} \\
& \sim \frac{\zeta(m+1)}{m-s} \frac{n^{m-s}}{\log n}=\frac{\zeta(m+1)}{m-(s+1)+1} \frac{n^{m-(s+1)+1}}{\log n} .
\end{aligned}
$$

That is, equation (5). The theorem is proved.

If $s=m$ then we obtain the following corollary. 
Corollary 2.3 The following asymptotic formula holds

$$
F_{m}^{m}(n)=\sum_{k=1}^{n}\left(\frac{P(k)}{k}\right)^{m} \sim \zeta(m+1) \frac{n}{\log n} \quad(m \geq 1) .
$$

If $m=1$ then equation (15) becomes the Kemeny's formula (3).

Note that if $k$ is prime then we have $P(k)=k$. Consequently each prime contributes 1 to the sum $F_{m}^{m}(n)$ (see (15)).

Theorem 2.4 Let $m$ be an arbitrary but fixed positive integer. We have the following asymptotic formulae

$$
f_{m}^{s}(n)=\sum_{k=1}^{n} \frac{p(k)^{m}}{k^{s}} \sim \frac{1}{m-s+1} \frac{n^{m-s+1}}{\log n} \quad(s=0,1, \ldots, m) .
$$

Proof. If $s=0$ then the theorem is true (see [2]). Now, the proof is the same as Theorem 2.2. The theorem is proved.

If $s=m$ then we obtain the following corollary.

Corollary 2.5 The following asymptotic formula holds

$$
f_{m}^{m}(n)=\sum_{k=1}^{n}\left(\frac{p(k)}{k}\right)^{m} \sim \frac{n}{\log n} \quad(m \geq 1) .
$$

Note that if $k$ is prime then we have $p(k)=k$. Consequently each prime contributes 1 to the sum $f_{m}^{m}(n)$ (see (16)).

ACKNOWLEDGEMENTS. The author is very grateful to Universidad Nacional de Luján.

\section{References}

[1] K. Alladi and P. Erdős, On an additive arithmetic function, Pacific Journal of Mathematics, 71 (1977), 275 - 294.

[2] R. Jakimczuk, A note on sums of greatest (least) prime factors, International Journal of Contemporary Mathematical Sciences, 8 (2013), 423 432 .

[3] J. Kemeny, Largest prime factor, Journal of Pure and Applied Algebra, 89 (1993), 181 - 186.

[4] J. Rey Pastor, P. Pi Calleja y C. A. Trejo, Análisis Matemático, Kapelusz, 1969.

Received: April 5, 2014 